\title{
Follow Up of COVID-19 Features in Recovered Adults without Comorbidities- Persistent Symptoms and Lab-Abnormalities
}

Julian Varghese ( $\square$ medizininformatik@uni-muenster.de )

University of Münster https://orcid.org/0000-0002-7206-3719

Sarah Sandmann

University of Münster https://orcid.org/0000-0002-5011-0641

Richard Vollenberg

University Hospital Münster

Kevin Ochs

University Hospital Münster

Inga Schrempf

University Hospital Münster

Christopher Froemmel

University Hospital Münster

Martin Dugas

University of Münster https://orcid.org/0000-0001-9740-0788

Hartmut Schmidt

University Hospital Münster

Phil Tepasse

University Hospital Münster

\section{Article}

Keywords: health care, COVID-19, recovered patients, comorbidities

Posted Date: November 30th, 2020

DOl: https://doi.org/10.21203/rs.3.rs-116030/v1

License: (c) (i) This work is licensed under a Creative Commons Attribution 4.0 International License.

Read Full License 


\section{Abstract}

Background: With increasing numbers of patients recovering from COVID-19, there is increasing evidence for persisting symptoms and the need for follow-up studies.

Methods: This retrospective study included patients without comorbidities, who recovered from COVID-19 and attended an outpatient clinic at a university hospital for follow-up care and potential convalescent plasma donation. Network analysis was applied to visualize symptom combinations and persistent symptoms. Comprehensive lab-testing was ascertained at each follow-up to analyze differences regarding patients with vs without persistent symptoms.

Results: 116 patients were included, age range was 18-69 years (median: 41) with follow-ups ranging from 22 to 102 days. The three most frequent persisting symptoms were Fatigue (54\%), Dyspnea (29\%) and Anosmia (25\%). Lymphopenia was present in 13 of 112 (12\%) cases. Five of 35 cases (14\%) had Lymphopenia in the later follow-up range of 80-102 days. Serum IgA concentration was the only lab parameter with significant difference between patients with vs without persistent symptoms with reduced serum IgA concentrations in the patient cohort of persisting symptoms $(p=0.0219)$.

Conclusion: Lymphopenia persisted in a noticeable percentage of recovered patients. Moreover, higher serum IgA concentration is associated with a positive effect regarding symptom persistence.

\section{Introduction}

COVID-19 (Coronavirus disease 2019) disease has spread as pandemic, causing more than 1 million global deaths as of Novemer $2020^{1}$ and shows a multifaceted condition with various symptoms due to various organ manifestations ${ }^{2-5}$. There is also an increasing number of recovered patient cases, which can provide new essential insights on the disease course. While the clinical feature characterization of COVID-19 is well-studied for the disease onset ${ }^{3,6-8}$, follow-up information on persisting symptoms and lab findings is scarce ${ }^{9}$. In addition, most work on symptomatology has not studied clusters of cooccurring symptoms in a single patient. Indeed, a systematic review by Struyf et al ${ }^{10}$ found that combinations of symptoms were not assessed in a single study. However, as newer publications are quickly emerging, we found one study on frequent symptoms clusters ${ }^{11}$.

This work is a retrospective evaluation of university hospital data of patients, who recovered from COVID19 and were screened for convalescent plasma donation eligibility. Since patients with comorbidities were excluded, our study provides insights on the healthy population, in which COVID-19 disease course is not well-studied.

Standardized symptom characterization report forms and all free-text elements of discharge letters were added for analyses. This facilitates the extraction of infection-related symptoms, which are not explicitly listed in structured forms. Moreover, details on persisting symptoms and duration to symptom-free phase could be sufficiently extracted in almost all of the cases. As a simple, but highly expressive visualization 
technique, network analysis illustrates clusters of symptoms and persisting symptoms in one graphbased plot. Blood sampling enabled comprehensive lab-data including whole blood analysis, serum chemistry and immunoglobulins. The combination of detailed symptom duration documentation and raw lab-data enables deep analyses of lab differences - also within the physiological range - in patients with versus without persisting symptoms. By doing this, the study evaluated lab abnormalities including but not limited to general infection parameters, in particular lymphopenia, being discussed as a hot-topic and prognostic factor in disease onset ${ }^{12-14}$.

\section{Methods}

\section{Setting}

This is a single center retrospective study in the University Hospital of Münster in Germany. Patients who recovered after confirmed diagnosis of COVID-19 were invited upon an open public call in the Münsterland region of Germany. The primary purpose of this call was to find eligible patients for a plasma donation study (local ethics committee approval: AZ 2020-210-f-S) ${ }^{15}$. All included patients provided informed consent for scientific analyses. Figure 1 illustrates the screening process and data collection. Patients with comorbidities or active infection symptoms within the last four weeks were excluded. Final assessment was carried out via outpatient visit at the University Hospital with medical history taking and lab testing. From this stage, further patients were excluded from this study if comorbidities were deemed to be existing or if there was missing information on symptom onset or symptom duration.

\section{Data extraction}

Symptoms were obtained from structured clinical forms and discharge letters. Attending physicians were required to document symptoms onset, symptoms duration and if symptoms are currently existing. Symptoms were defined to be persistent if they endured for at least 28 days from symptom onset. Single symptoms and possible combinations were extracted, but not the duration of each single symptom as this was not documented consistently.

Lab values and corresponding reference ranges were queried from the laboratory information system. Discharge letters were examined by a physician with infection-related symptoms being mapped to medical concepts of the standardized forms or added as new symptoms. Symptoms being highly similar were summarized to one medical concept.

\section{Data Analysis}

Network analysis of symptoms was conducted using the R-package igraph $1.2^{16}$ to show frequency and graph-based co-occurrence of onset-symptoms and persisting symptoms. Descriptive statistics were 
calculated for patient cohort characteristics. Age and gender were compared in the two cohorts with vs without persistent symptoms using 2-tailed t-tests (alpha=0.05) and Fisher's exact test, respectively. Lab data was summarized with descriptive statistics and proportions below and above reference ranges. In addition, lab values were compared between the two cohorts using 2-tailed t-tests (alpha $=0.05$; adjustment for multiple testing using Bonferroni correction). Calculations were conducted in $\mathrm{R}$, version 4.03.

\section{Results}

\section{Patient Cohort}

After participation in the online-survey, 122 patients were screened eligible and attended the outpatient clinic for final assessment during June and September 2020. Six patients were then excluded due to insufficient information on symptom duration or comorbidities that were found to be existent $(n=116)$. The age range was 18-69 years, with median of 41 years (IQR 30-54). Table 1 summarizes further cohort details. Comparing the two patient cohorts with $(n=92)$ vs without persistent symptoms $(n=24)$, no significant difference can be observed regarding age (t-test, $p=0.2497)$ and gender (Fisher's exact test, $p=0.3415$ ).Follow-up ranged from 22 to 102 days. Figure 2 illustrates follow-up times from symptom onset and symptom durations of all patients.

Table 1

Demographic and Clinical Characteristics of patients $(n=116)$.

\begin{tabular}{|ll|}
\hline Patient Characteristics $(\mathbf{n}=\mathbf{1 1 6})$ & Value \\
\hline Age, median (IQR), y & $41(31-55)$ \\
\hline Female sex, no. (\%) & $17(15 \%)$ \\
\hline Follow-up time, median (IQR), d & $66(44-82)$ \\
\hline Positive SARS-CoV2- antibody testing, no. (\%) & $70(80 \%)$ \\
\hline Requiring Hospital admission no. (\%) & $10(9 \%)$ \\
\hline With Persistent Symptoms, No. (\%) & $24(21 \%)$ \\
\hline • Age, median (IQR), y & $44(34-57)$ \\
\hline • Female sex, No. (\%) & $5(21 \%)$ \\
\hline Without Persisting Symptoms & $92(79 \%)$ \\
\hline • Age, median (IQR), y & $41(30-54)$ \\
\hline • Female sex, No. (\%) & $12(13 \%)$ \\
\hline
\end{tabular}

Symptom Analyses 
After extraction of infection-related concepts in the discharge letters, the concept "Reduced Physical Resilience" was subsumed to "Fatigue". Myalgia and signs of arthralgia were subsumed to Myalgia. Tinnitus was added as new symptom as it did not occur in the structured standard. Figure 3 shows the network analysis of onset and persisting symptoms. The five most frequent onset symptoms were Cough (50\%), Anosmia (47\%), Fatigue (45\%), Fever (42\%), Myalgia (28\%) and Headache (28\%). Among patients with persisting symptoms $(n=24)$, the three most frequent persisting symptoms were Fatigue $(n=13$, $54 \%$, $\min =28 d, \max \geq 94 d)$, Dyspnea $(29 \%, \min =25 d, \max \geq 102 d)$ and Anosmia $(25 \%, \min =41 \mathrm{~d}$, $\max$ $\geq 91 \mathrm{~d}$ ). A web-based platform was established to upload anonymous symptoms data to generate and reproduce network analysis based on own or other cohorts ${ }^{17}$.

\section{Lab Abnormalities}

Table 2 summarizes the outcome of all lab measurements, which were available in more than $95 \%$ of all patient cases. Increased values above the reference range can be observed in more than $10 \%$ of patients for Creatine Kinase, Glucose, GOT, GPT, Potassium, LDH and Platelets count. Decreased values can be observed for eGFR and absolute Lymphocyte count. Lymphopenia was present in 13 of 112 (12\%) cases. These cases had results below the lab-specific reference range of $1.26-3.35 \times 10^{9} / \mathrm{L}$ (follow-up range: 45 102d, median: 70d). In the late follow-up range of 80-102 days, lymphopenia was still present in five of 35 cases (14\%, follow-up range: $82-102 d$, median: $83 d$ ). Figure 4 details lymphocyte count over all followup times for all cases. 
Table 2

Lab values in alphabetical order for all measures with at least $95 \%$ availability among all participants. The columns named below and above represent the percentage of patients deviating from the reference range, which encompasses $95 \%$ of the healthy population. Comparison PS = Comparison of lab-values in the two cohorts: With vs without persistent symptoms.

\begin{tabular}{|c|c|c|c|c|c|c|c|}
\hline \multirow[t]{3}{*}{ Lab-Value (Unit) } & \multicolumn{3}{|l|}{ All } & \multicolumn{4}{|c|}{ Comparison PS vs N-PS } \\
\hline & $\begin{array}{l}\text { Normal } \\
\text { range \% }\end{array}$ & Below\% & $\begin{array}{l}\text { Above } \\
\%\end{array}$ & $\begin{array}{l}\text { PS, } \\
\text { mean }\end{array}$ & $\begin{array}{l}\text { N-PS, } \\
\text { mean }\end{array}$ & $\begin{array}{l}\mathrm{p}- \\
\text { value }\end{array}$ & $\begin{array}{l}\text { Adjusted } \\
\text { p-value }\end{array}$ \\
\hline & & & & (SD) & (SD) & & \\
\hline Albumin (g/dL) & 98,23 & 0,00 & 1,77 & $\begin{array}{l}4.54 \\
(0.31)\end{array}$ & $\begin{array}{l}4.59 \\
(0.22)\end{array}$ & 0,4709 & 1 \\
\hline $\begin{array}{l}\text { Alkal. } \\
\text { phosphatase(U/L) }\end{array}$ & 98,23 & 1,77 & 0,00 & $\begin{array}{l}62.87 \\
(15.72)\end{array}$ & $\begin{array}{l}62.83 \\
(15.91)\end{array}$ & 0,9922 & 1 \\
\hline Antithrombin (\%) & 99,11 & 0,89 & 0,00 & $\begin{array}{l}98.57 \\
(7.55)\end{array}$ & $\begin{array}{l}97.69 \\
(8.68)\end{array}$ & 0,6321 & 1 \\
\hline $\begin{array}{l}\text { Basophile abs. } \\
(1000 / \mu \mathrm{L})\end{array}$ & 93,75 & 0,89 & 5,36 & $\begin{array}{l}0.04 \\
(0.01)\end{array}$ & $\begin{array}{l}0.04 \\
(0.02)\end{array}$ & 0,1819 & 1 \\
\hline Basophile rel. (\%) & 94,64 & 0,89 & 4,46 & $\begin{array}{l}0.62 \\
(0.29)\end{array}$ & $\begin{array}{l}0.74 \\
(0.34)\end{array}$ & 0,1079 & 1 \\
\hline $\begin{array}{l}\text { Bilirubin total } \\
(\mathrm{mg} / \mathrm{dL})\end{array}$ & 95,58 & 0,00 & 4,42 & $\begin{array}{l}0.73 \\
(0.59)\end{array}$ & $\begin{array}{l}0.56 \\
(0.24)\end{array}$ & 0,1788 & 1 \\
\hline CRP (mg/dL) & 94,69 & 0,00 & 5,31 & $\begin{array}{l}0.41 \\
(0.04)\end{array}$ & $\begin{array}{l}0.41 \\
(0.06)\end{array}$ & 0,7412 & 1 \\
\hline Creatinine (mg/dL) & 100,00 & 0,00 & 0,00 & $\begin{array}{l}0.91 \\
(0.16)\end{array}$ & $\begin{array}{l}0.92 \\
(0.14)\end{array}$ & 0,7813 & 1 \\
\hline Creatine kinase (U/L) & 66,37 & 0,00 & 33,63 & $\begin{array}{l}121.78 \\
(64.42)\end{array}$ & $\begin{array}{l}173.76 \\
(139.93)\end{array}$ & 0,0110 & 0,5598 \\
\hline $\begin{array}{l}\text { eGFR - CKD EPI } \\
(\mathrm{mL} / \mathrm{min})\end{array}$ & 75,22 & 24,78 & 0,00 & $\begin{array}{l}93.57 \\
(4.91)\end{array}$ & $\begin{array}{l}93.82 \\
(5)\end{array}$ & 0,8246 & 1 \\
\hline Total Protein (g/dL) & 97,35 & 1,77 & 0,88 & $\begin{array}{l}7.38 \\
(0.34)\end{array}$ & $\begin{array}{l}7.38 \\
(0.36)\end{array}$ & 0,9302 & 1 \\
\hline $\begin{array}{l}\text { Eosinoph. abs. } \\
(1000 / \mu \mathrm{L})\end{array}$ & 94,64 & 2,68 & 2,68 & $\begin{array}{l}0.14 \\
(0.08)\end{array}$ & $\begin{array}{l}0.15 \\
(0.12)\end{array}$ & 0,5812 & 1 \\
\hline $\begin{array}{l}\text { Eosinophile relative } \\
\text { (\%) }\end{array}$ & 95,54 & 1,79 & 2,68 & $\begin{array}{l}2.49 \\
(1.56)\end{array}$ & $\begin{array}{l}2.68 \\
(2.02)\end{array}$ & 0,6246 & 1 \\
\hline $\begin{array}{l}\text { Erythrocytes/RBC } \\
(\text { Mio./ } \mu \mathrm{L})\end{array}$ & 87,50 & 2,68 & 9,82 & $\begin{array}{l}4.92 \\
(0.45)\end{array}$ & $5(0.41)$ & 0,4013 & 1 \\
\hline Ferritin $(\mu \mathrm{g} / \mathrm{L})$ & 83,19 & 7,08 & 9,73 & $\begin{array}{l}191.48 \\
(185.22)\end{array}$ & $\begin{array}{l}177.03 \\
(140.19)\end{array}$ & 0,7294 & 1 \\
\hline
\end{tabular}




\begin{tabular}{|c|c|c|c|c|c|c|c|}
\hline \multirow[t]{3}{*}{ Lab-Value (Unit) } & \multicolumn{3}{|l|}{ All } & \multicolumn{4}{|c|}{ Comparison PS vs N-PS } \\
\hline & $\begin{array}{l}\text { Normal } \\
\text { range \% }\end{array}$ & Below\% & $\begin{array}{l}\text { Above } \\
\%\end{array}$ & $\begin{array}{l}\text { PS, } \\
\text { mean }\end{array}$ & $\begin{array}{l}\text { N-PS, } \\
\text { mean }\end{array}$ & $\begin{array}{l}\mathrm{p}- \\
\text { value }\end{array}$ & $\begin{array}{l}\text { Adjusted } \\
\text { p-value }\end{array}$ \\
\hline & & & & (SD) & (SD) & & \\
\hline Fibrinogen (mg/dL) & 95,58 & 0,88 & 3,54 & $\begin{array}{l}311.65 \\
(78.52)\end{array}$ & $\begin{array}{l}294.34 \\
(48.33)\end{array}$ & 0,3220 & 1 \\
\hline Gamma-GT (U/L) & 93,81 & 0,00 & 6,19 & $\begin{array}{l}33.04 \\
(25.52)\end{array}$ & $\begin{array}{l}30.23 \\
(22.04)\end{array}$ & 0,6319 & 1 \\
\hline Glucose (mg/dL) & 88,60 & 0,88 & 10,53 & $\begin{array}{l}90 \\
(17.68)\end{array}$ & $\begin{array}{l}90.85 \\
(21.71)\end{array}$ & 0,8461 & 1 \\
\hline GOT/AST. (U/L) & 81,42 & 0,00 & 18,58 & $\begin{array}{l}28.09 \\
(5.42)\end{array}$ & $\begin{array}{l}29.1 \\
(8.7)\end{array}$ & 0,4892 & 1 \\
\hline GPT/ALT. (U/L) & 78,76 & 0,00 & 21,24 & $\begin{array}{l}30.3 \\
(18.04)\end{array}$ & $\begin{array}{l}31.02 \\
(13.38)\end{array}$ & 0,8595 & 1 \\
\hline Hematokrit (\%) & 93,75 & 6,25 & 0,00 & $\begin{array}{l}42.95 \\
(3.22)\end{array}$ & $\begin{array}{l}43.1 \\
(2.83)\end{array}$ & 0,8372 & 1 \\
\hline Hemoglobin (g/dL) & 93,75 & 5,36 & 0,89 & $\begin{array}{l}14.53 \\
(1.23)\end{array}$ & $\begin{array}{l}14.64 \\
(1.1)\end{array}$ & 0,6949 & 1 \\
\hline $\lg A(m g / d L)$ & 92,98 & 1,75 & 5,26 & $\begin{array}{l}172.17 \\
(57.95)\end{array}$ & $\begin{array}{l}231.29 \\
(97.25)\end{array}$ & 0,0004 & 0,0219 \\
\hline $\lg G(\mathrm{mg} / \mathrm{dL})$ & 98,25 & 0,00 & 1,75 & $\begin{array}{l}1143.2 \\
(185.03)\end{array}$ & $\begin{array}{l}1113.07 \\
(193.5)\end{array}$ & 0,4937 & 1 \\
\hline $\operatorname{lgM}(\mathrm{mg} / \mathrm{dL})$ & 87,72 & 6,14 & 6,14 & $\begin{array}{l}146.26 \\
(192.42)\end{array}$ & $\begin{array}{l}102.78 \\
(73.43)\end{array}$ & 0,2979 & 1 \\
\hline INR (ratio) & 100,00 & 0,00 & 0,00 & $\begin{array}{l}0.99 \\
(0.05)\end{array}$ & $\begin{array}{l}0.98 \\
(0.05)\end{array}$ & 0,7382 & 1 \\
\hline $\begin{array}{l}\text { Immat. Granulocytes } \\
(1000 / \mu \mathrm{L})\end{array}$ & 99,11 & 0,00 & 0,89 & $\begin{array}{l}0.02 \\
(0.01)\end{array}$ & $\begin{array}{l}0.02 \\
(0.01)\end{array}$ & 0,2752 & 1 \\
\hline Interleukin.6 (pg/mL) & 99,12 & 0,00 & 0,88 & $\begin{array}{l}2.09 \\
(0.52)\end{array}$ & $\begin{array}{l}2.15 \\
(1.1)\end{array}$ & 0,6824 & 1 \\
\hline LDH (U/L) & 68,14 & 0,88 & 30,97 & $\begin{array}{l}226.74 \\
(46.82)\end{array}$ & $\begin{array}{l}210.77 \\
(39.87)\end{array}$ & 0,1431 & 1 \\
\hline Leukocytes (Tsd./ $\mu \mathrm{L})$ & 92,86 & 7,14 & 0,00 & $\begin{array}{l}6.11 \\
(1.36)\end{array}$ & $\begin{array}{l}5.77 \\
(1.32)\end{array}$ & 0,2826 & 1 \\
\hline Lipase (U/L) & 93,81 & 0,00 & 6,19 & $\begin{array}{l}40.13 \\
(12.79)\end{array}$ & $\begin{array}{l}35.32 \\
(14.33)\end{array}$ & 0,1252 & 1 \\
\hline $\begin{array}{l}\text { Lymphocytes abs. } \\
(1000 / \mu \mathrm{L})\end{array}$ & 86,61 & 11,61 & 1,79 & $\begin{array}{l}1.8 \\
(0.57)\end{array}$ & $\begin{array}{l}1.81 \\
(0.54)\end{array}$ & 0,9501 & 1 \\
\hline
\end{tabular}




\begin{tabular}{|c|c|c|c|c|c|c|c|}
\hline \multirow[t]{3}{*}{ Lab-Value (Unit) } & \multicolumn{3}{|l|}{ All } & \multicolumn{4}{|c|}{ Comparison PS vs N-PS } \\
\hline & $\begin{array}{l}\text { Normal } \\
\text { range \% }\end{array}$ & Below\% & $\begin{array}{l}\text { Above } \\
\%\end{array}$ & $\begin{array}{l}\text { PS, } \\
\text { mean }\end{array}$ & $\begin{array}{l}\text { N-PS, } \\
\text { mean }\end{array}$ & $\begin{array}{l}\mathrm{p}- \\
\text { value }\end{array}$ & $\begin{array}{l}\text { Adjusted } \\
\text { p-value }\end{array}$ \\
\hline & & & & (SD) & (SD) & & \\
\hline $\begin{array}{l}\text { Lymphocytes relative } \\
(\%)\end{array}$ & 91,07 & 6,25 & 2,68 & $\begin{array}{l}29.62 \\
(7.16)\end{array}$ & $\begin{array}{l}31.77 \\
(7.34)\end{array}$ & 0,2084 & 1 \\
\hline $\mathrm{MCH}(\mathrm{pg})$ & 91,96 & 6,25 & 1,79 & $\begin{array}{l}29.61 \\
(1.66)\end{array}$ & $\begin{array}{l}29.31 \\
(1.59)\end{array}$ & 0,4460 & 1 \\
\hline $\mathrm{MCHC}(\mathrm{g} / \mathrm{dL})$ & 83,93 & 3,57 & 12,50 & $\begin{array}{l}33.82 \\
(1.01)\end{array}$ & $\begin{array}{l}33.96 \\
(0.93)\end{array}$ & 0,5573 & 1 \\
\hline MCV (fL) & 91,07 & 8,93 & 0,00 & $\begin{array}{l}87.58 \\
(3.85)\end{array}$ & $\begin{array}{l}86.32 \\
(4.07)\end{array}$ & 0,1771 & 1 \\
\hline $\begin{array}{l}\text { Monocytes abs. } \\
(1000 / \mu \mathrm{L})\end{array}$ & 95,54 & 2,68 & 1,79 & $\begin{array}{l}0.53 \\
(0.18)\end{array}$ & $\begin{array}{l}0.51 \\
(0.14)\end{array}$ & 0,5755 & 1 \\
\hline $\begin{array}{l}\text { Monozyten relative } \\
(\%)\end{array}$ & 97,32 & 1,79 & 0,89 & $\begin{array}{l}8.89 \\
(2.54)\end{array}$ & $\begin{array}{l}8.97 \\
(1.91)\end{array}$ & 0,8864 & 1 \\
\hline Sodium (mmol/L) & 100,00 & 0,00 & 0,00 & $\begin{array}{l}140.74 \\
(1.68)\end{array}$ & $\begin{array}{l}140.74 \\
(1.68)\end{array}$ & 0,7060 & 1 \\
\hline $\begin{array}{l}\text { Neutrophiles abs. } \\
(1000 / \mu \mathrm{L})\end{array}$ & 95,54 & 4,46 & 0,00 & $\begin{array}{l}3.6 \\
(1.06)\end{array}$ & $\begin{array}{l}3.26 \\
(1.05)\end{array}$ & 0,1686 & 1 \\
\hline $\begin{array}{l}\text { Neutrophiles relative } \\
(\%)\end{array}$ & 93,75 & 3,57 & 2,68 & $\begin{array}{l}58.4 \\
(6.65)\end{array}$ & $\begin{array}{l}55.84 \\
(8.55)\end{array}$ & 0,1309 & 1 \\
\hline $\begin{array}{l}\text { Part. Thrombopl. } \\
\text { Time (s) }\end{array}$ & 91,15 & 0,88 & 7,96 & $\begin{array}{l}34.13 \\
(2.6)\end{array}$ & $\begin{array}{l}34.19 \\
(3.01)\end{array}$ & 0,9263 & 1 \\
\hline Platelets $(1000 / \mu \mathrm{L})$ & 83,04 & 6,25 & 10,71 & $\begin{array}{l}265.3 \\
(71.97)\end{array}$ & $\begin{array}{l}236.55 \\
(48.63)\end{array}$ & 0,0809 & 1 \\
\hline Potassium (mmol/L) & 74,34 & 4,42 & 21,24 & $\begin{array}{l}4.3 \\
(0.67)\end{array}$ & $\begin{array}{l}4.35 \\
(0.62)\end{array}$ & 0,7593 & 1 \\
\hline $\begin{array}{l}\text { Procalcitonin } \\
(\mathrm{ng} / \mathrm{mL})\end{array}$ & 100,00 & 0,00 & 0,00 & $\begin{array}{l}0.05 \\
(0.02)\end{array}$ & $\begin{array}{l}0.05 \\
(0.03)\end{array}$ & 0,4861 & 1 \\
\hline $\begin{array}{l}\text { Pseudocholinesterase } \\
\text { (U/L) }\end{array}$ & 100,00 & 0,00 & 0,00 & $\begin{array}{l}8493.1 \\
(1884.5)\end{array}$ & $\begin{array}{l}8642.5 \\
(1476.9)\end{array}$ & 0,7264 & 1 \\
\hline Sodium (mmol/L) & 100,00 & 0,00 & 0,00 & $\begin{array}{l}140.74 \\
(1.68)\end{array}$ & $\begin{array}{l}140.74 \\
(1.68)\end{array}$ & 0,7060 & 1 \\
\hline Thrombin time (s) & 100,00 & 0,00 & 0,00 & $\begin{array}{l}17.52 \\
(0.79)\end{array}$ & $\begin{array}{l}17.19 \\
(0.7)\end{array}$ & 0,0748 & 1 \\
\hline $\mathrm{TSH}(\mu \mathrm{U} / \mathrm{mL})$ & 100,00 & 0,00 & 0,00 & $\begin{array}{l}1.4 \\
(0.69)\end{array}$ & $\begin{array}{l}1.5 \\
(0.63)\end{array}$ & 0,5134 & 1 \\
\hline
\end{tabular}




\begin{tabular}{|llllllll|}
\hline Lab-Value (Unit) & All & \multicolumn{5}{c|}{ Comparison PS vs N-PS } \\
\cline { 2 - 8 } & $\begin{array}{l}\text { Normal } \\
\text { range \% }\end{array}$ & Below\% & $\begin{array}{l}\text { Above } \\
\%\end{array}$ & $\begin{array}{l}\text { PS, } \\
\text { mean } \\
\text { (SD) }\end{array}$ & $\begin{array}{l}\text { N-PS, } \\
\text { mean } \\
\text { (SD) }\end{array}$ & $\begin{array}{l}\text { p- } \\
\text { value }\end{array}$ & $\begin{array}{l}\text { Adjusted } \\
\text { p-value }\end{array}$ \\
\hline Urea $(\mathrm{mg} / \mathrm{dL})$ & 99,12 & 0,00 & 0,88 & $\begin{array}{l}13.43 \\
(3.3)\end{array}$ & $\begin{array}{l}14.07 \\
(3.45)\end{array}$ & 0,4223 & 1 \\
\hline
\end{tabular}

After adjusting for multiple testing for all lab measurements, IgA was the only parameter - also among other Immunoglobulins - that remained significantly different between patients with and without persistent symptoms (see Table 2 ). It was significantly reduced in patients with persisting symptoms $(p=$ 0.0219).

\section{Discussion}

This study found that a noticeable amount of COVID-19-recovered patients still had $(n=24,21 \%)$ persisting symptoms. In $13 \%$ of the cases they persisted for 60 days and longer. The three most frequent ones were Fatigue, Anosmia and Dyspnea. These results are similar to existing follow-up studies: A postacute-follow-up study in Italy by Carfi et al. $2020^{9}$ evaluated Fatigue and Dyspnea but not Anosmia as persistent symptoms (mean follow-up 60.3 days). A follow-up study by Carvalho-Schneider et al. 202018 reported the identical set of our most common persisting symptoms (follow-up at day 30 and 60, noncritical COVID-19 patients). Comparing to a study by Garrigues et al. $2020{ }^{19}$ with a mean of 110,9 days, Fatigue and Dyspnea were the most common, followed by Loss of Memory. These findings support a common symptom set regardless of patient case severity. However, as with many symptom-related infection studies, signs of neuro-degenerative or mental health disorders could be under-reported. They were not documented in our study as they were not reported by the patients and not part of the structured forms.

Regarding duration of persistent symptoms, our results are significantly different: The post-acute setting in Carfi et al. observed $87.2 \%$ of patients having persistent symptom with mean follow-up of around 60 days. Calvao-Schneider et al. $2020^{18}$ reported that $66 \%$ of patients experienced symptoms at day 6018 . The difference should be explained by our comorbidity-free and relatively young population (median age: $41 \mathrm{ys}$ ). Moreover, the majority of the population in Calvao-Schneider et al. had an initial hospitalization rate of $35 \%$ vs. $9 \%$ in our study.

The main strength of our study is the integration of detailed symptoms duration and raw lab-data, which enabled not only assessment of out of range values but also differences within the physiological range. The study results show that some of the infection-related lab values remained out of range during followup. In particular, lymphopenia - being frequently discussed as a result of direct or indirect viral interactions with lymphocytes ${ }^{12-14}$ - remained in a considerable part of our study population, even beyond 90 days of follow-up (see Fig. 4). To the best of our knowledge, this is the first COVID-19-related 
study on the duration of lymphopenia along with other routine lab values going beyond 30 days. Further follow-up studies should assess lymphopenia with additional lab analysis to understand the pathogenetic details of lymphocyte-virus interaction.

The comparison of patients with vs without persisting symptoms did not show any differences of lab values except for one: serum concentration of total IgA antibodies. In comparison, there were no significant changes regarding total IgG, IgM and all other lab-tests in Table 2. Secretory IgA is an

important factor of mucosal immunity for neutralization of toxins and pathogenic microbes ${ }^{20,21}$. While a high level of serum IgA and SARS-CoV-2-specific IgA on diagnosis can initially correlate with disease severity 22,23 , our findings suggest that high levels during or after recovery is associated with a decrease in persistent symptoms. In this regard, IgA may play a more important role than other immunoglobulin types. As this study only reports on lab-value correlations, further research can complement our findings regarding causality.

The principal limitation of this study is the retrospective single-center design with symptom assessment not being objective and being prone to recall bias. Moreover, the different follow-up times also lead to different time distances between last day of symptoms and the actual lab-assessment. As some labvalues return to normal levels over a certain time, some lab tests and association with symptom persistence could have faded out and thus failed to show significance in our analysis.

\section{Declarations}

\section{Author Contributions}

JV established the study design, preprocessed the data and wrote the manuscript. SS conducted data analyses. RV, KO, IMS, CF and PRT were involved in data acquisition and preparation. MD and HHS supervised the project. All authors significantly contributed to the manuscript and approved for publication.

\section{Competing Interests}

The authors declare no competing interests.

\section{References}

1. Dong E, Du H, Gardner L. An interactive web-based dashboard to track COVID-19 in real time. The Lancet infectious diseases. 2020;20(5):533-534.

2. Zhou F, Yu T, Du R, Fan G, Liu Y, Liu Z, et al. Clinical course and risk factors for mortality of adult inpatients with COVID-19 in Wuhan, China: a retrospective cohort study. Lancet. 2020 28;395(10229):1054-62. 
3. Kumar A, Arora A, Sharma P, Anikhindi SA, Bansal N, Singla V, et al. Clinical Features of COVID-19 and Factors Associated with Severe Clinical Course: A Systematic Review and Meta-Analysis. SSRN. 2020 Apr 21;3566166.

4. Menter T, Haslbauer JD, Nienhold R, Savic S, Hopfer H, Deigendesch N, et al. Postmortem examination of COVID-19 patients reveals diffuse alveolar damage with severe capillary congestion and variegated findings in lungs and other organs suggesting vascular dysfunction. Histopathology. 2020;77(2):198-209.

5. Gupta A, Madhavan MV, Sehgal K, Nair N, Mahajan S, Sehrawat TS, et al. Extrapulmonary manifestations of COVID-19. Nature medicine. 2020;26(7):1017-1032.

6. Wu Z, McGoogan JM. Characteristics of and Important Lessons From the Coronavirus Disease 2019 (COVID-19) Outbreak in China: Summary of a Report of 72314 Cases From the Chinese Center for Disease Control and Prevention. JAMA. 2020 Apr 7;323(13):1239-42.

7. Li J, Huang DQ, Zou B, Yang H, Hui WZ, Rui F, et al. Epidemiology of COVID-19: A systematic review and meta-analysis of clinical characteristics, risk factors, and outcomes. Journal of Medical Virology [Internet]. [cited 2020 Sep 15];n/a(n/a). Available from:

https://onlinelibrary.wiley.com/doi/abs/10.1002/jmv.26424

8. Li L, Huang T, Wang Y, Wang Z, Liang Y, Huang T, et al. COVID-19 patients' clinical characteristics, discharge rate, and fatality rate of meta-analysis. Journal of Medical Virology. 2020;92(6):577-83.

9. Carfi A, Bernabei R, Landi F. Persistent symptoms in patients after acute COVID-19. Jama. 2020;324(6):603-605.

10. Struyf T, Deeks JJ, Dinnes J, Takwoingi Y, Davenport C, Leeflang MM, et al. Signs and symptoms to determine if a patient presenting in primary care or hospital outpatient settings has COVID-19 disease. Cochrane Database Syst Rev. 2020 07;7:CD013665.

11. Docherty AB, Harrison EM, Green CA, Hardwick HE, Pius R, Norman L, et al. Features of 20133 UK patients in hospital with COVID-19 using the ISARIC WHO Clinical Characterisation Protocol: prospective observational cohort study. bmj. 2020;369.

12. Terpos E, Ntanasis-Stathopoulos I, Elalamy I, Kastritis E, Sergentanis TN, Politou M, et al. Hematological findings and complications of COVID-19. American Journal of Hematology. 2020;95(7):834-47.

13. Tan L, Wang Q, Zhang D, Ding J, Huang Q, Tang Y-Q, et al. Lymphopenia predicts disease severity of COVID-19: a descriptive and predictive study. Signal Transduction and Targeted Therapy. 2020 Mar $27 ; 5(1): 1-3$.

14. Zhao Q, Meng M, Kumar R, Wu Y, Huang J, Deng Y, et al. Lymphopenia is associated with severe coronavirus disease 2019 (COVID-19) infections: A systemic review and meta-analysis. International Journal of Infectious Diseases. 2020 Jul 1;96:131-5.

15. Dugas M, Grote-Westrick T, Vollenberg R, Lorentzen E, Brix T, Schmidt $H$, et al. Less severe course of COVID-19 is associated with elevated levels of antibodies against seasonal human coronaviruses OC43 and HKU1 (HCoV OC43, HCoV HKU1). medRxiv. 2020 Oct 14;2020.10.12.20211599. 
16. https://igraph.org/ [Internet]. Available from: https://igraph.org/

17. Sarah Sandmann. Network analysis of onset and persistent symptoms in COVID 19 patients. [Internet]. [cited 2020 Nov 18]. Available from: https://sand-imi-unimuenster.shinyapps.io/COVIDSymptomNetwork/

18. Carvalho-Schneider C, Laurent E, Lemaignen A, Beaufils E, Bourbao-Tournois C, Laribi S, et al. Followup of adults with non-critical COVID-19 two months after symptoms' onset. Clin Microbiol Infect. 2020 Oct 5;

19. Garrigues E, Janvier P, Kherabi Y, Le Bot A, Hamon A, Gouze H, et al. Post-discharge persistent symptoms and health-related quality of life after hospitalization for COVID-19. J Infect [Internet]. 2020 Aug 25 [cited 2020 Oct 22]; Available from: https://www.ncbi.nlm.nih.gov/pmc/articles/PMC7445491/

20. Renegar KB, Small PA. Passive transfer of local immunity to influenza virus infection by IgA antibody. The Journal of Immunology. 1991 Mar 15;146(6):1972-8.

21. Macpherson AJ, McCoy KD, Johansen F-E, Brandtzaeg P. The immune geography of IgA induction and function. Mucosal Immunology. 2008 Jan;1(1):11-22.

22. Hasan Ali O, Bomze D, Risch L, Brugger SD, Paprotny M, Weber M, et al. Severe COVID-19 is associated with elevated serum IgA and antiphospholipid IgA-antibodies. Clin Infect Dis [Internet]. [cited 2020 Oct 22]; Available from: https://academic.oup.com/cid/advancearticle/doi/10.1093/cid/ciaa1496/5913451

23. Dahlke C, Heidepriem J, Kobbe R, Santer R, Koch T, Fathi A, et al. Distinct early IgA profile may determine severity of COVID-19 symptoms: an immunological case series. medRxiv. $2020 \mathrm{Apr}$ 17;2020.04.14.20059733.

\section{Figures}




\section{Münsterland Region, Germany}

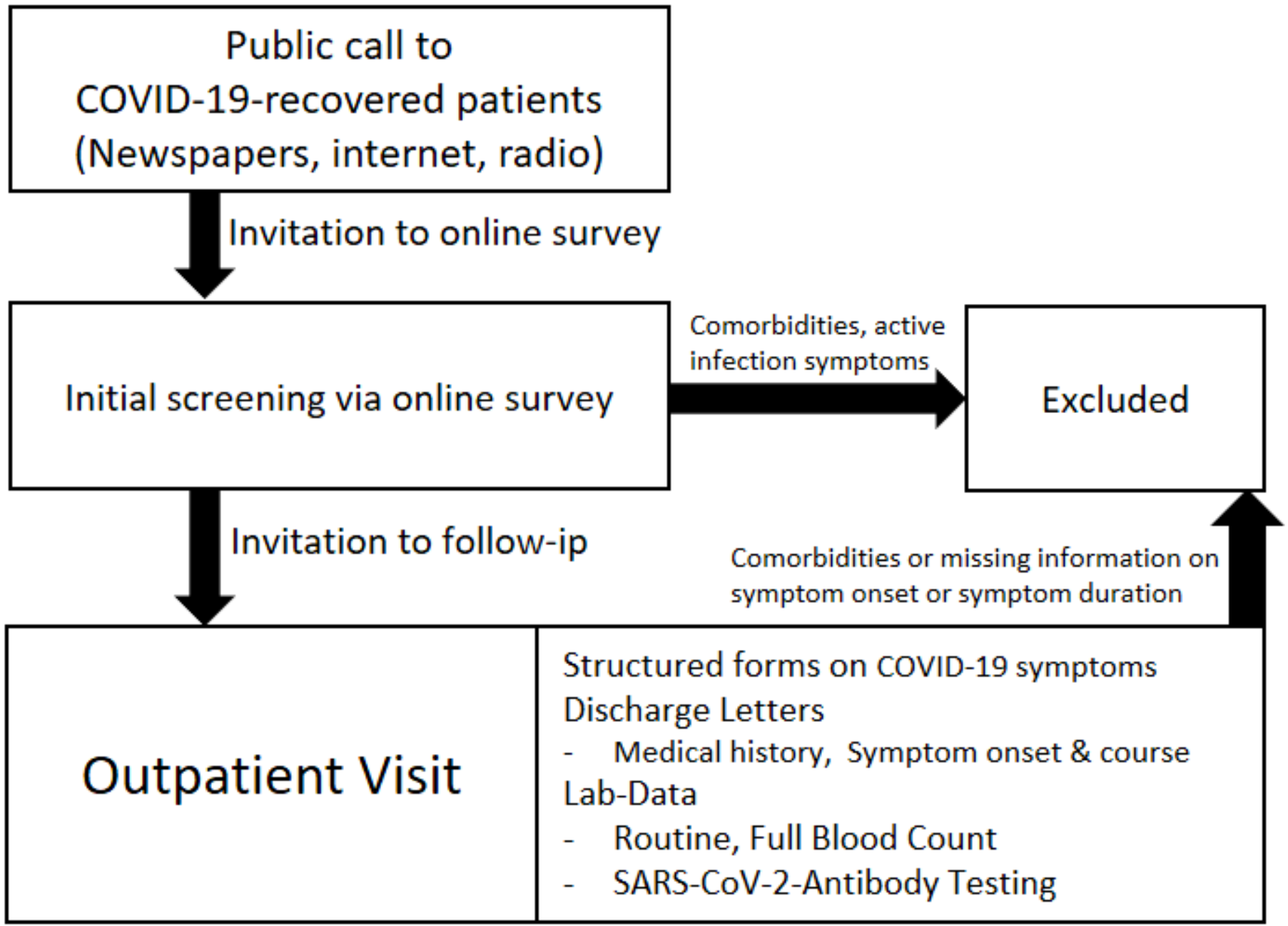

\section{University Hospital Münster}

Figure 1

Inclusion of study population.

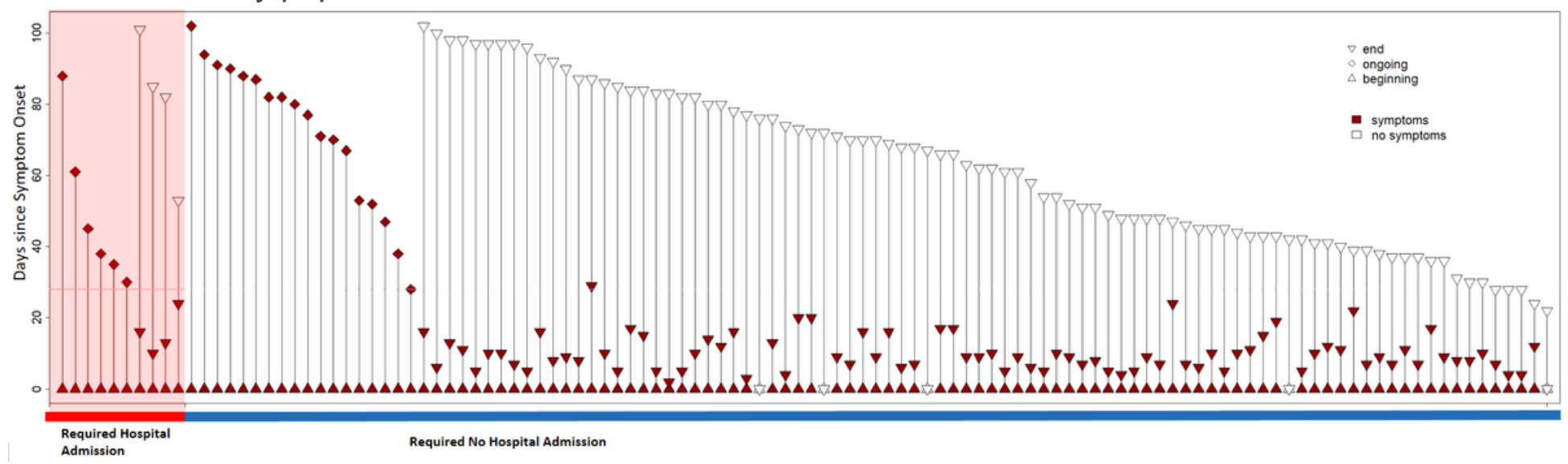

Figure 2 
Follow-up day and symptom duration for each patient. Grey horizontal line represents threshold for persistent symptoms (28 days). Five patients were asymptomatic (symptom start and end at day 0 ).

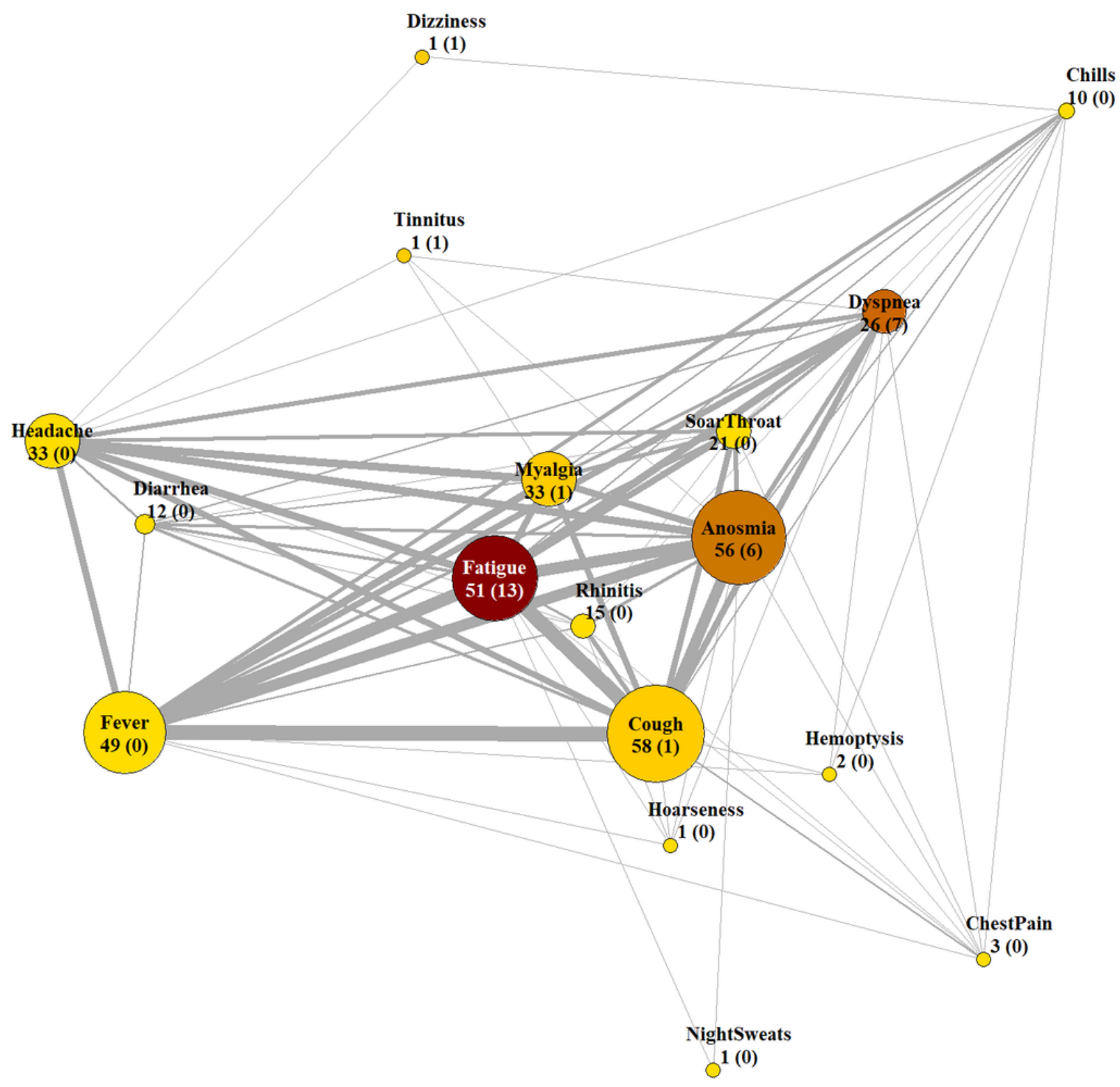

\section{Figure 3}

Network analyses of symptom nodes. Node size and node number correspond to the number of patients having that onset symptom. The number in parentheses corresponds to persistent symptoms, which is also indicated by the redness of the symptom node. Thickness of grey edges corresponds co-occurrence of onset symptoms. 
Absolute lymphocyte count (reference $1.26-3.35 \times 10^{\wedge}$ 9/L)

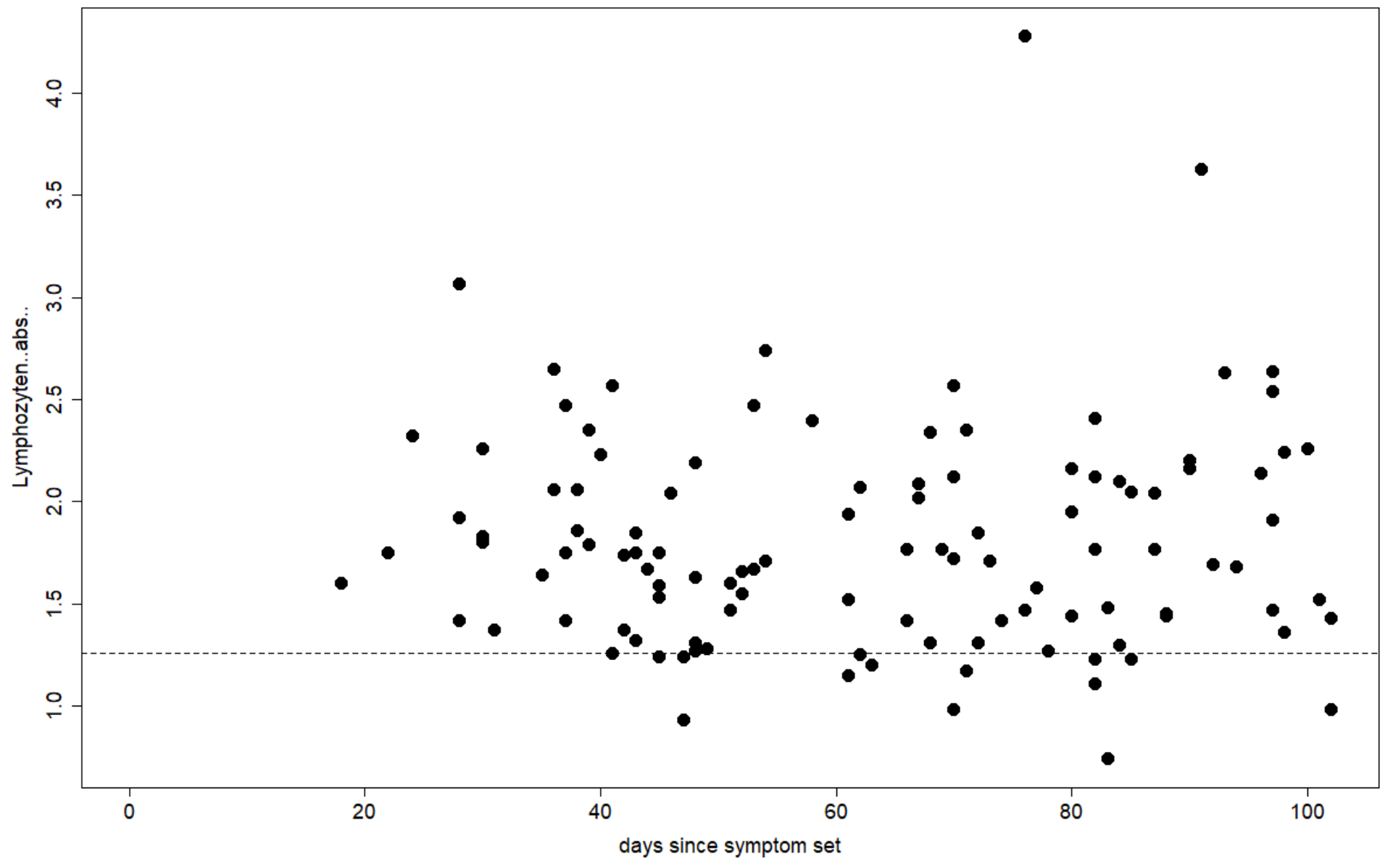

Figure 4

Absolute lymphocyte count over time. Dashed line represents lab-specific lower limit (1.26x 109/L) 